\title{
Protect your patients and colleagues from the impact of flu
}

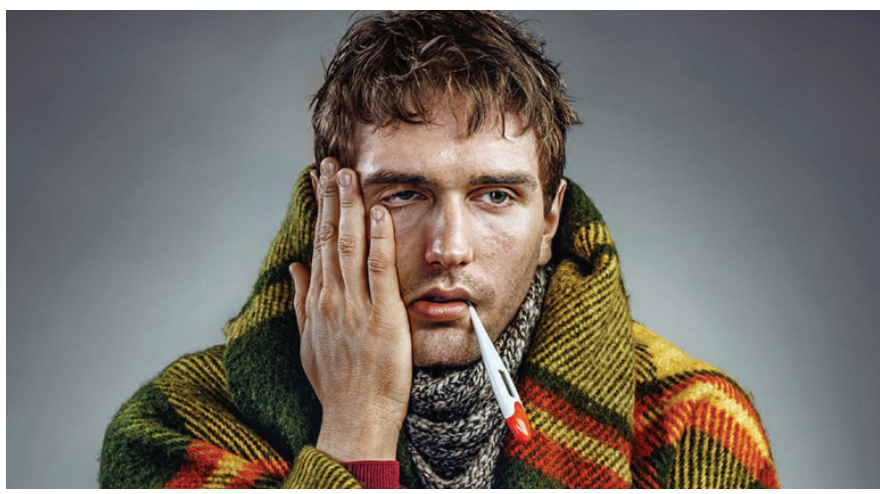

This year's influenza warning comes with extra caution - Australia recently witnessed the worst flu outbreak for many years and the deadly H3N2 strain could be on its way to the UK.

\section{For visibly whiter teeth}

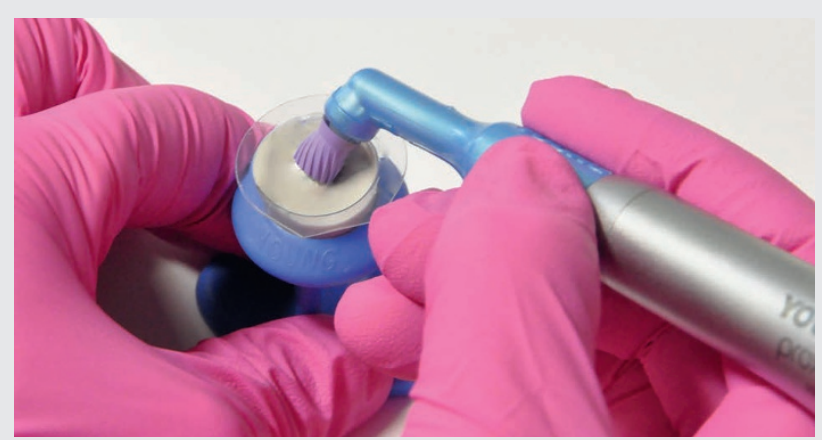

Young Dental presents a range of new pastes for professional teeth cleaning. They remove discoloration, achieve significant teeth whitening, and impress with their remarkable efficiency. The $\mathrm{pH}$-neutral pastes are less abrasive than similar products, so have a particularly gentle feel, and are vegan as well as sugar-, lactose- and gluten-free.

The 'mint' and 'berry' varieties are supplied in single doses, and come with a handy finger-ring holder for free. The hygienic disposable packaging minimises the risk of cross-contamination. A fluoride-free polishing paste with fine granulation and a stain remover in medium granulation with $1.23 \%$ fluoride are available. To reduce splatter, Young Dental has developed an especially adhesive consistency for the pastes.

The sugar substitute xylitol reduces the development of plaque as well as the production of tooth-damaging acids, and has a proven cariostatic and even anti-cariogenic effect. Another component is natron, which shortens the cleaning time and enhaances teeth whitening. Furthermore, it improves the buffering capacity of saliva and removes harmful microorganisms in plaque. At the same time, thanks to the acid-neutralising effect, the growth of caries bacteria is slowed.

Young Innovations is a leading developer, manufacturer and retailer of high-quality dental products. The American company has its headquarters in Chicago; the centre of European operations is in Heidelberg, Germany. www.youngdental.eu.
To protect your patients and colleagues from the potential impact of flu, it's crucial to ensure strict hand hygiene and effective disinfection of all surfaces and inanimate objects.

The Steri-7 Xtra range of multipurpose biocidal products from Initial Medical deactivate $99.9999 \%$ of pathogens, including influenza A and various other viruses, infections, fungi, spores and yeasts. Non-toxic and non-irritant, the high level surface disinfectants feature 'Reactive Barrier Technology' that uses a specialised micro emulsion for long-term protection of up to 72 hours after application.

Fully compliant with all relevant legislation, Steri-7 Xtra is a must for all dental practices this flu season.

There is a very real possibility of an influenza outbreak this winter make sure you're prepared.

For further information please visit www.initial.co.uk/medical or call 08708504045 .

\section{Protect the next generation}

Are your patients aware of the importance of orofacial protection during sport?

Mouth guards have moved on from their image of obtrusive items that impede speech. Offer custom-made mouth guards from Saber Protect and you will allow your patients to enjoy excellent protection, comfort and fit.

Saber Protect mouth guards are unique to the individual and have been carefully designed to give range of incredible benefits. These include:

- Layered protection and impact dispersion, which might prevent temporomandibular joint dislocation as well as tooth-related injury

- A perfect fit, so breathing and speaking is not affected and the device does not fall out easily at the moment of impact

- Sports specific, to meet the needs of every patient.

Dental injuries are the most common type of orofacial trauma sustained during sport, but they are easily prevented with a custom-made mouth guard. If a patient wears one habitually, they could avoid the devastating impact of an injury.

Find out more about Saber Protect custom-made mouth guards to offer your patients superior protection. This service will not only enhance the reputation of your practice, but could be a valuable revenue stream too.

Saber Protect custom-made mouth guards are fabricated by CosTech Dental Laboratory. For more information visit www.custom-mouth-guards.com. 